\title{
Microshaping metal surfaces by wave-directed self-organization
}

\author{
Pil J. Yoo, S. Young Park, S. Joon Kwon, Kahp Y. Suh, and Hong H. Lee ${ }^{a)}$ \\ School of Chemical Engineering, Seoul National University, Seoul, 151-744, Korea
}

(Received 25 July 2003; accepted 6 October 2003)

\begin{abstract}
Self-organization in the shaping of a metal surface is dictated by an internal wave that selects the type of modes from an externally imposed periodic pattern. An elastomeric mold, when placed on a thin bilayer of metal on polymer and heated, provides periodic nodes that give rise to a periodic wave of harmonic series. The internal wave in the bilayer selects the type, number of harmonic modes, and the fractional magnitude that each allowed harmonic mode contributes to the overall surface shape, thereby permitting shape engineering of the metal surface. (C) 2003 American Institute of Physics. [DOI: 10.1063/1.1630377]
\end{abstract}

The phenomenon of surface wrinkling has received much attention because of its significance in thin film science and industry. ${ }^{1-4}$ Controlling the wrinkling so as to produce a desired pattern or shape is a key issue in the surface wrinkling, and yet only recently have there been studies on utilizing the wrinkling for the purpose of generating regular patterns. ${ }^{5-7}$ In the thin bilayer of a metal on a polymer being considered, wrinkles are generated upon heating above the glass transition temperature $\left(T_{g}\right)$ of the polymer to relieve the stress generated due to a difference in the thermal expansion coefficients of the two layers. It has been shown that when a patterned polydimethylsiloxane (PDMS) mold is placed on the bilayer and the system is heated, the externally imposed pattern of the mold causes the wrinkles to be shaped as a simple sinusoidal wave. ${ }^{8}$

A specific question raised in this work is how an externally imposed periodic wave causes the surface of a bilayer to evolve into different shapes. As shown in Fig. 1(a), the usual isotropic wrinkles are created in the absence of an external mold. When a mold with a periodic pattern is placed onto the bilayers with a different intrinsic wavelength, however, the bilayers are made to self-organize into the various ordered shapes shown in Figs. 1(b) through 1(e) even with the same mold. This wave-directed self-organization does not lead to a surface with a simple sinusoidal structure or shape.

For the experiments, a monodispersed polystyrene (PS, $M_{n}=4.04 \times 10^{6}, M_{w} / M_{n}=1.05$, Polymer Source, Inc.) with toluene as the solvent was spin-coated onto the silicon substrate to various thicknesses ranging from 150 to $800 \mathrm{~nm}$. For the metal, we used thermally deposited aluminum, ranging in thickness from 30 to $100 \mathrm{~nm}$. The PDMS molds were prepared by mixing a siloxane base oligomer and a curing agent (Sylgard 184, Dow Corning) in the ratio of 15 to 1 by weight, pouring the mixture onto the master molds and then curing at $50^{\circ} \mathrm{C}$ for $12 \mathrm{~h}$. For the wave-directed selforganization, the bilayer samples and the PDMS molds were separately heated to a temperature slightly below $T_{g}$ of PS to avoid the undue stress generated during heating. The PDMS mold was then placed on the sample and the temperature was

\footnotetext{
a) Author to whom correspondence should be addressed; electronic mail: honghlee@snu.ac.kr
}

raised to $130{ }^{\circ} \mathrm{C}$, which is well above the $T_{g}$ of PS $\left(105^{\circ} \mathrm{C}\right)$. Typical annealing time was $12 \mathrm{~h}$. It was then allowed to cool down to room temperature and the mold was removed. The resulting surface structures were examined by atomic force microscopy (AFM, Digital Instruments, Dimension 3100) in the contact mode. Figure 2(a) is an optical micrograph of the metal surface formed by wave-directed self-organization.

Although the isotropic wrinkles lack directional order [Fig. 1(a)], they have a certain intrinsic wavelength associated with them as shown in the fast Fourier transform image. This wavelength can be determined theoretically in an a priori manner. ${ }^{8}$ When a patterned PDMS mold is placed on the bilayer, it makes a strong conformal contact with the underlying metal surface, which makes the edges of the pattern act as nodes. These nodes dictate the nodal condition to be satisfied; that is, creation of nodal waves within the period of

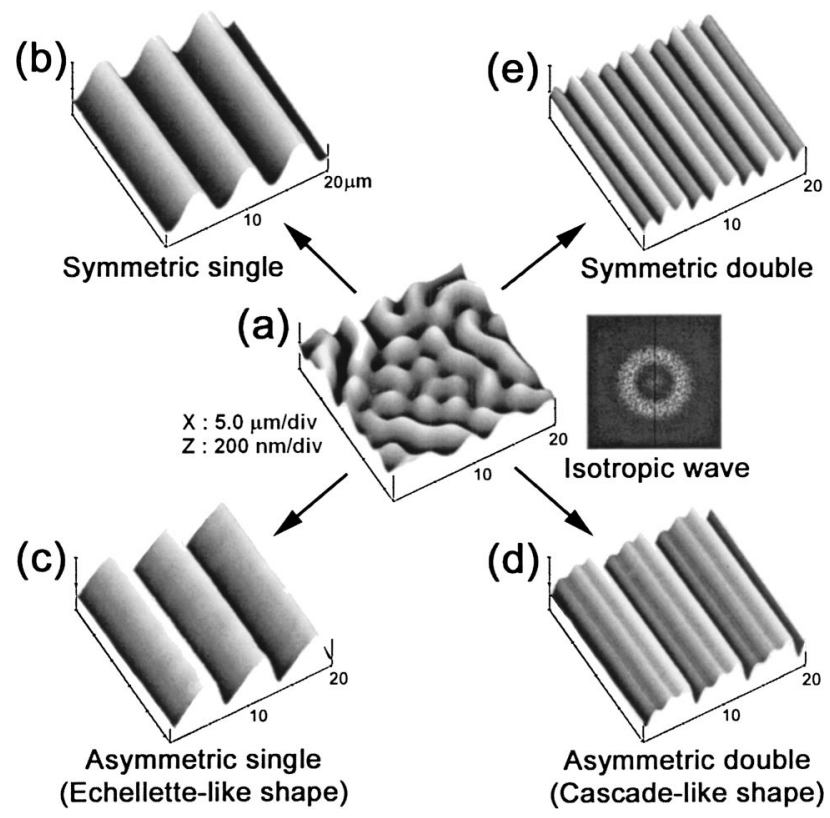

FIG. 1. Three-dimensional AFM images of the metal surface formed by the self-organization. For all the shapes formed, the same PDMS mold (3 $\mu \mathrm{m}$ line-and-space) was used. (a) Isotropic waves in the absence of the mold. (b) Symmetric single mode $\left(t_{m}=80 \mathrm{~nm}, t_{p}=520 \mathrm{~nm}, \lambda_{i}=4.68 \mu \mathrm{m}\right)$. (c) Asymmetric double mode $\left(t_{m}=60 \mathrm{~nm}, t_{p}=600 \mathrm{~nm}, \lambda_{i}=3.92 \mu \mathrm{m}\right)$. (d) Asymmetric double mode $\left(t_{m}=50 \mathrm{~nm}, t_{p}=350 \mathrm{~nm}, \lambda_{i}=2.96 \mu \mathrm{m}\right)$. (e) Symmetric double mode $\left(t_{m}=30 \mathrm{~nm}, t_{p}=220 \mathrm{~nm}, \lambda_{i}=1.68 \mu \mathrm{m}\right)$. 
(a)

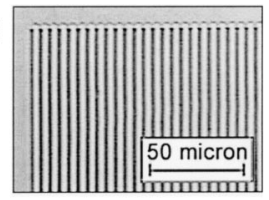

(c)

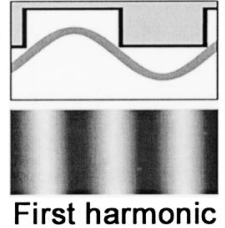

(b)

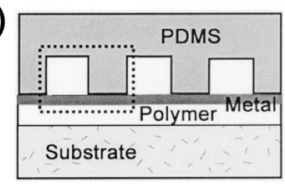

(d)

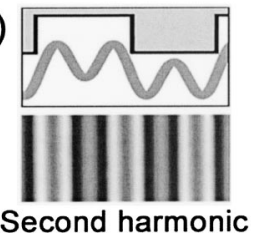

FIG. 2. (a) Optical micrograph of line-and-space metal surface formed by the self-organization. (b) A schematic of the experimental setup. The dotted box denotes the repeating unit structure of self-organization. (c) First harmonic case $\left(\lambda_{i}\right.$ is almost the same as $\left.\lambda_{m}\right)$. (d) Second-harmonic case $\left(\lambda_{i}\right.$ is smaller than $\lambda_{m}$ ). Waves are allowed as odd harmonics to satisfy the symmetry condition in the confined unit structure.

the mold pattern [see Fig. 2(b)]. The question is how these nodal waves created by the externally imposed wave interact with the internal, intrinsic wave.

In the shaping of the metal surface, the nodal condition must be satisfied and the shape is simply the result of a linear combination of the nodal waves. If we normalize the wave profile with respect to the amplitude $\epsilon$ and utilize the symmetric nature of the mold, the normalized wave profile $w(x)$ can be written as

$$
w(x)=\epsilon \sum_{n=1}^{\infty} \frac{f_{n}}{(2 n-1)} \sin \left[(2 n-1) k_{m} x\right], \quad n=1,2,3, \ldots,
$$

where $x$ is the axis of the wave, $f_{n}$ is the fractional contribution from the $n$th harmonic, and $k_{m}$ is the mold wave number given by $2 \pi / \lambda_{m}$, where $\lambda_{m}$ is the mold wavelength. Odd harmonics were chosen to satisfy the symmetry condition in the repeating unit structure consisting of one void and one contact, as shown in Figs. 2(c) and 2(d).

The resulting harmonic conditions are not at the global minimum free energy corresponding to the intrinsic wave number. In the absence of the global minimum, the system keeps adding harmonics to the harmonic series starting with the first mode until adding an additional harmonic results in an increase of the free energy. Let $F\left(k_{m}\right)$ be the free energy of the first harmonic $(n=1$ and $2 n-1=1)$. If the next higher mode ( $n=2$ and $2 n-1=3)$ is to be included in the harmonic series, its free energy $F\left(3 k_{m}\right)$ has to be at least equal to $F\left(k_{m}\right)$ to reach a minimum:

$$
F\left(k_{m}\right)=F\left(3 k_{m}\right) \text {. }
$$

The free energy of the bilayer ${ }^{8,9}$ is given by the sum of the bending energy of the metal layer ${ }^{10}$ and the elastic deformation energy of the underlying polymer layer: ${ }^{11}$

$$
F(k)=\left[\frac{E_{m} k^{2} t_{m}^{3}}{12\left(1-\nu_{m}^{2}\right)}+\frac{E_{p}}{k^{4} t_{p}^{3}}+\frac{2 E_{p}}{3 k}\right] \frac{(\epsilon k)^{2}}{4},
$$

where $t$ is the thickness, $E$ is Young's modulus, the subscripts $m$ and $p$ are for the metal and the polymer, respectively, $k$ is the wave number, and $\nu_{m}$ is the Poisson ratio of the metal. Equation (2) has been solved with the aid of Eq.

(3) for $k_{i} / k_{m}$ that satisfies Eq. (2). For all the experimental for $k_{i} / k_{m}$ that satisfies Eq. (2). For all the experimental pression is
Downloaded 20 Nov 2003 to 18.42 .2 .210 . Redistribution subject to AlP license conditions, the ratio was calculated to be nearly constant at $1.6,{ }^{12}$ which means that the value of the mold wavelength in excess of the intrinsic wavelength is allowed up to $60 \%$ for the first mode, and the second mode comes in when it exceeds the ratio. For the third mode, $n=3$ and $2 n-1=5$, and therefore the second mode is allowed up to the mold wavelength that satisfies the condition given by $F\left(3 k_{m}\right)$ $=F\left(5 k_{m}\right)$. As was done for the first mode, one can get the value of 3.82 as the ratio of wavelengths. Similarly, the third mode is allowed up to 5.88, the fourth mode is allowed up to 7.93 , and so on.

One notable finding in our experimental results is that asymmetric waves form as shown in Figs. 1(c) and 1(d), which deviate from the symmetric harmonic series that consists of odd harmonic waves. This symmetry breaking for the double mode originates from the system's desire to minimize its free energy at the risk of an uneven contact of PDMS mold's pattern, allowing even harmonic waves, in effect selecting nodes from within two periods rather than one period. An even second mode will lower the free energy as long as its free energy is smaller than the free energy associated with the odd second mode; that is, $F\left(2 k_{m}\right) \leqslant F\left(3 k_{m}\right)$. This condition, when solved for the wavelength ratio, yields a value of 2.43, which gives the range to which an even second mode is allowed. Therefore, the range of the ratio $\lambda_{m} / \lambda_{i}$, within which an asymmetric double mode results, is from 1.60 to 2.43 , which in turn gives the range $2.43<\lambda_{m} / \lambda_{i}<3.80$ as the symmetric double-mode range. Other asymmetric modes are not observed for higher modes because the free energy per unit wave shows little difference whether it is a symmetric or an asymmetric wave, making the higher asymmetric modes irrelevant. The accommodation of the even harmonic gives rise to echellette-like [Fig. 1(c)] or cascade-like [Fig. 1(d)] shape, which is useful for optical applications, as in shallow gratings. ${ }^{13}$

Shown in Fig. 3 are the AFM images of surface profiles that were generated experimentally by the wave-directed self-organization. All the experimental results agree with the theoretical results given earlier that are based on the free energy consideration. For a given bilayer, the surface shapes are entirely determined by the ratio of the mold and intrinsic wavelengths. From top down in any given column in the figure, one can see the shape change with the increase in the mold wavelength $\left(\lambda_{m}\right)$ for a given intrinsic wavelength $\left(\lambda_{i}\right)$. Conversely, from left to right in any given row, one can also observe the change with increasing intrinsic wavelength for a given mold. For the case of $\lambda_{m}=6 \mu \mathrm{m}$, for example, one can generate symmetric double mode, asymmetric double mode, and symmetric single mode wave simply by varying the film thickness.

Now that the number of harmonics and their types that are to be included in the harmonic series are determined, it only remains for the engineering of the metal surface shape to resolve the fractional weighting $f_{n}$. The expression for the free energy of the self-organized wave can be derived in a straightforward manner, although cumbersome. ${ }^{10,11}$ The ex-

to AlP license or copyright, see http://ojps.aip.org/aplo/aplcr.jsp 
(a)

(b)

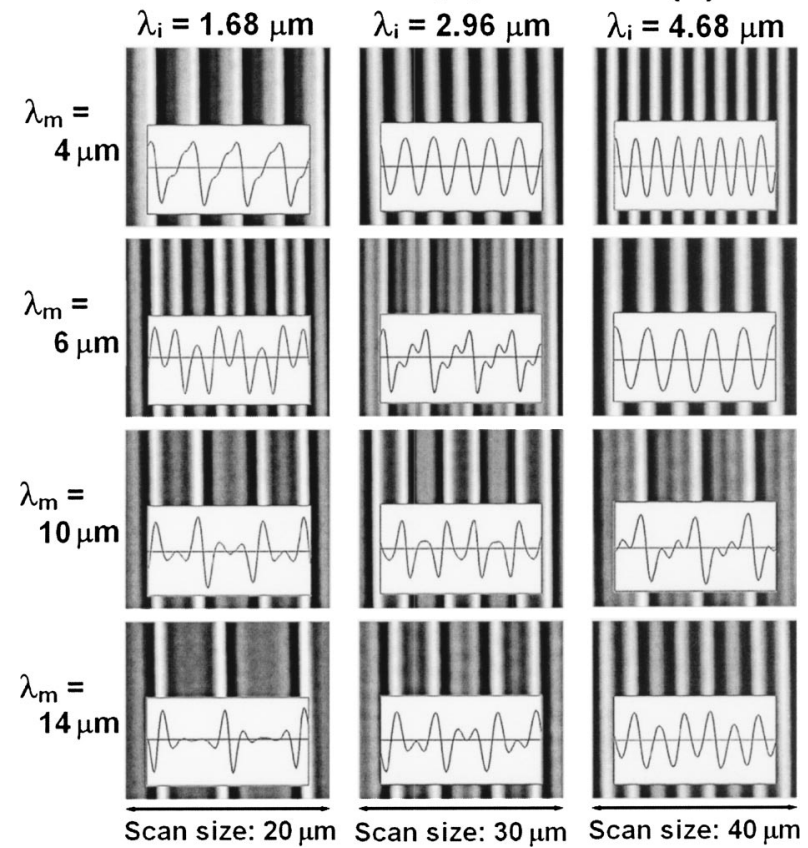

FIG. 3. Two-dimensional AFM micrographs and the corresponding surface profiles (insets) of the self-organized metal surface. (a) Column for $\lambda_{i}$ of $1.68 \mu \mathrm{m}$. The shape changes from asymmetric double mode $\left(\lambda_{m}\right.$ $=4 \mu \mathrm{m})$, to symmetric double mode $\left(\lambda_{m}=6 \mu \mathrm{m}\right)$, to symmetric triple mode $\left(\lambda_{m}=10 \mu \mathrm{m}\right)$, and to symmetric quadruple mode $\left(\lambda_{m}=14 \mu \mathrm{m}\right)$. (b) Column for $\lambda_{i}$ of $2.96 \mu \mathrm{m}$. The shape changes from symmetric single mode $\left(\lambda_{m}=4 \mu \mathrm{m}\right)$, to asymmetric double $\left(\lambda_{m}=6 \mu \mathrm{m}\right)$, to symmetric double $\left(\lambda_{m}=10 \mu \mathrm{m}\right)$, and to symmetric triple $\left(\lambda_{m}=14 \mu \mathrm{m}\right)$. (c) Column for $\lambda_{i}$ of $4.68 \mu \mathrm{m}$. The shape changes from symmetric single mode $\left(\lambda_{m}=4\right.$ and $6 \mu \mathrm{m})$, to asymmetric double $\left(\lambda_{m}=10 \mu \mathrm{m}\right)$, and to symmetric double $\left(\lambda_{m}=14 \mu \mathrm{m}\right)$.

$$
\begin{aligned}
& F_{n}=\frac{\left(\epsilon_{0} k_{i}\right)^{2}}{4} \sum_{n=1}^{n} f_{n}^{2} C_{n}, \\
& C_{n}=\frac{(2 n-1)^{3} E_{m} k_{m}^{2} t_{m}^{3}}{12\left(1-\nu_{m}^{2}\right)}+\frac{E_{p}}{(2 n-1)^{3} k_{m}^{4} t_{p}^{3}}+\frac{2 E_{p}}{3 k_{m}},
\end{aligned}
$$

where $\epsilon_{0}$ is the average amplitude of the intrinsic buckling wave. Under the condition of constant external strain, minimization of the free energy $F_{n}$ with respect to $f_{n}$ yields the relative fraction. ${ }^{14}$ Typical examples of the comparison between theory and experiment are shown in Fig. 4. The figure shows that the theoretical shape follows quite well the metal surface profile obtained by AFM measurement. The metal surface shapes as represented by the harmonics up to the second mode [Fig. 4(a)] and the third mode [Fig. 4(b)] shown here indicate that the theory can capture the shape and the peaks quite well. With all the terms in the harmonic series determined, one can simply engineer the shape of the metal surface.

The ability to tailor the shape of a solid surface would find potential applications in many areas. In particular, the (a)
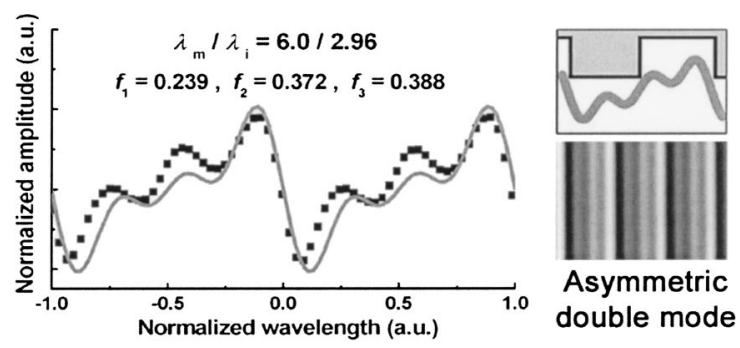

Asymmetric double mode

(b)
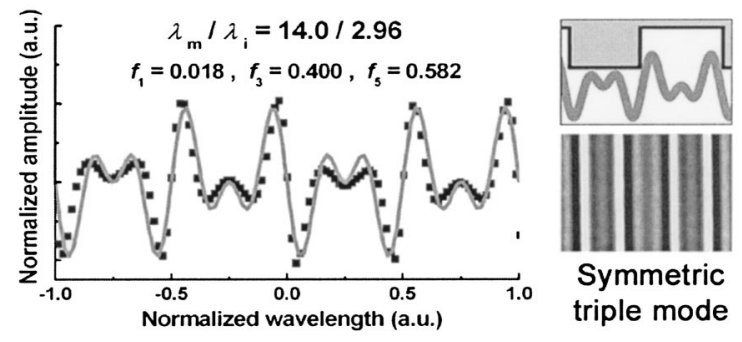

FIG. 4. Comparison between experimental results (dots converted from the AFM profile) and theoretical results (solid gray line). (a) Asymmetric double mode. (b) Symmetric triple mode.

advantage of tunability for an optical surface can be utilized as a promising method for making an optical device with other techniques that have been proposed. ${ }^{15,16}$

This work was supported by the Brain Korea 21 Project in 2003.

${ }^{1}$ S. P. Timoshenko and J. M. Gere, Theory of Elastic Stability (McGrawHill, New York, 1961).

${ }^{2}$ H. G. Allen, Analysis and Design of Structural Sandwich Panels (Permagon, New York, 1969).

${ }^{3}$ N. Sridhar, D. J. Srolovitz, and Z. Suo, Appl. Phys. Lett. 78, 2482 (2001).

${ }^{4}$ R. Huang and Z. Suo, J. Appl. Phys. 91, 1135 (2002).

${ }^{5}$ N. Bowden, S. Brittain, A. G. Evans, J. W. Hutchinson, and G. M. Whitesides, Nature (London) 393, 146 (1998).

${ }^{6}$ N. Bowden, W. T. S. Huck, K. E. Paul, and G. M. Whitesides, Appl. Phys. Lett. 75, 2557 (1999).

${ }^{7}$ D. B. H. Chua, H. T. Ng, and S. F. Y. Li, Appl. Phys. Lett. 76, 721 (2000).

${ }^{8}$ P. J. Yoo, S. Y. Park, K. Y. Suh, and H. H. Lee, Adv. Mater. (Weinheim, Ger.) 14, 1383 (2002)

${ }^{9}$ J. Groenwold, Physica A 298, 32 (2001).

${ }^{10}$ L. D. Landau and E. M. Lifshitz, Theory of Elasticity (Pergamon, Oxford, 1970).

${ }^{11}$ G. H. Fredrickson, A. Ajdari, L. Leibler, and J. Carton, Macromolecules 25, 2882 (1992).

${ }^{12}$ This result has been obtained from the following relationship: $\left(k_{i} / k_{m}\right)$ $=\left(6 \times\left(1+\sqrt{1+32 A C / B^{2}}\right) /\left(1+\sqrt{1+71.1 A C / B^{2}}\right)\right)^{1 / 3}$, where $A$ $=E_{m} t_{m}^{3} / 12\left(1-v_{m}^{2}\right), B=2 E_{p} / 3, C=E_{p} / t_{p}^{3}$. The ratio $k_{i} / k_{m}$ has been calculated for $A C / B^{2}$ ranging from 1 to $10^{3}$.

${ }^{13}$ M. C. Hutley, Diffraction Gratings (Academic, New York, 1982).

${ }^{14}$ For example, the expression for triple-mode wave is as follows:

$$
f_{n}=\left[C_{o} C_{p}\left(1-\delta_{n o}\right)\left(1-\delta_{n p}\right)\left(1-\delta_{o p}\right)\right] / \sum_{q} C_{q} C_{n}\left(1-\delta_{n q}\right), \quad n, o, p, q=1,2,3,
$$

where $\delta_{i j}$ is the Kronecker delta.

${ }^{15}$ Y. Xia, E. Kim, X. Zhao, J. A. Rogers, M. Prentiss, and G. M. Whitesides, Science 273, 347 (1996).

${ }^{16}$ E. G. Loewen and E. Popov, Diffraction Gratings and Applications (Marcel Dekker, New York, 1997). 